\title{
Optimization and Prediction of Durability and Utility Features of Three-Layer Piezoelectric Transducers
}

\author{
G. MIECZKOWSKI \\ Białystok University of Technology, ul. Wiejska 45C, 15-351 Białystok, Poland, E-mail: g.mieczkowski@pb.edu.pl
}

cross $^{\text {ref }}$ http://dx.doi.org/10.5755/j01.mech.24.3.17953

\section{Introduction}

For the first time, piezoelectric effect was described by authors of paper [1]. They developed constitutive equations describing the relation between deflection and electric field occurring in a piezoelectric element. Practical use of this effect is attributed to Paul Langevin. In 1917, he designed a piezoelectric ultrasonic generator which then was used for submarine location. Today, many devices using this piezoelectric effect are manufactured. They are used in such branches as medicine, industry or transport $[2,3]$.

In most piezoelectric transducers measuring/control system usually has a layer structure what to some extent makes it difficult to determine their electromechanical characteristics and strength. Both the former and the latter aspect was dealt with by many scientists.

In order to determine electromechanical characteristics of a piezoelectric transducer (relation between deformation and applied load) adequate simultaneous equations should be defined and solved. These equations bound together geometrical properties, materials properties and physical parameters, such as force, deflection and electric field.

Formulation of such simultaneous equations is considerably difficult. Material and geometrical inhomogeneity of the transducer global structure and anisotropy of piezoelectric materials force the use of some simplifications. Electromechanical characteristics of piezoelectric transducers were dealt with by authors of papers [4-14]. Smits et al. [4] as first formed and solved physical equations for a transducer made of two layers of even thickness and length. Adopting similar assumptions - the same length of all layers in a transducer - authors of the paper [5] provided solutions for a three-layer transducer, and [6-9] - a multi-layer transducer. Electromechanical characteristics of transducers with layers of different length were dealt with in papers [10-14].

For strength aspects of piezoelectric transducers, when regarding transducer as a global layered (composite) structure, in design calculations it is necessary to include a presence of geometrical-material notches occurring at the interface. Such structural notches locally generate stress fields with large gradients, what in consequence initiates a fracture process in the plane of bonding particular layers. Description of stress fields occurring around the structural notch-tip area located on the interface of bi-material structure was dealt with by authors of papers [15-17]. However, in papers [18-20] qualitative and quantitative nature of singular stress fields generated by structural notches occurring in piezoelectric transducers was analysed.
In literature, it is rare to find test results that bring together the strength and usable characteristics of piezoelectric transducers. Therefore the main purpose of the this paper was to develop procedures for determination of such geometrical-material characteristics of piezoelectric transducers, for which both its strength and deformation conditions are optimal.

\section{Analytical and numerical solutions}

\subsection{Subject of research}

The paper deals with three-layer bender as presented in Fig. 1. In the transducer structure two piezoelectric components 1 and 3 and a non-piezoelectric element 2 can be distinguished.

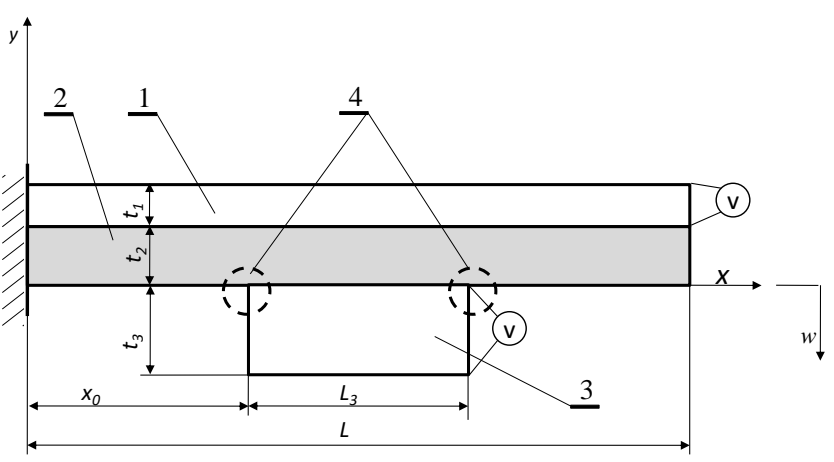

Fig. 1 Three-layer piezoelectric transducer:1-upper piezoelectric element, 2-non-piezoelectric element, 3-lower piezoelectric element, 4 -structural notch

It is assumed that upper piezoelectric layer 1 has the same length as element 2 . Length of component 3 (dimension $L_{3}$ ) is smaller than lengths of other layers dimension $L$ ). Layers have a different height $t_{i}$ and uniform thickness $b$. What is more, component 3 is located exactly in the middle of the transducer $-x_{0}=\left(L-L_{3}\right) / 2$. In analyses carried out it was assumed that Young's module for both piezoelectric elements is $E_{p}$, and for non-piezoelectric element $-E_{\mathrm{b}}$. Moreover, it was assumed that all components have the same Poisson's ratio $v$. Left side of the transducer is fixed, while the right side is freely movable (support conditions of a cantilever beam). The load comes from the applied electric voltage $V$ generating bending moment induced by a piezoelectric effect (occurring in piezoelectric components).

Purpose of tests carried out was to determine an optimum relation between stiffness and geometric characteristics of individual components of piezoelectric transducer, for which deflection of the right end of the transducer $w$ would be the greatest at the greatest resistance to fracture at the same time. As mentioned earlier, the point of fracture 
process initiation is at the tip of the structural notch 4 that is formed by lower piezoelectric layer and non-piezoelectric element.

For testing three optimization parameters were assumed, including:

1) $e=\frac{E_{b}}{E_{p}}-$ relative stiffness;

2) $t=\frac{t_{3}}{t_{1}}-$ relative height;

3) $\quad l=\frac{L}{L_{3}}-$ relative length.

Table 1

Cases of variability of optimization parameters obtained based on Box-Behnken plan

\begin{tabular}{|c|c|c|c|}
\hline Case no. & $e$ & $t$ & $l$ \\
\hline 1 & 1.00 & 0.50 & 0.50 \\
\hline 2 & 1.00 & 1.25 & 0.20 \\
\hline 3 & 1.00 & 1.25 & 0.80 \\
\hline 4 & 1.00 & 2.00 & 0.50 \\
\hline 5 & 10.50 & 0.50 & 0.20 \\
\hline 6 & 10.50 & 0.50 & 0.80 \\
\hline 7 & 10.50 & 1.25 & 0.50 \\
\hline 8 & 10.50 & 2.00 & 0.20 \\
\hline 9 & 10.50 & 2.00 & 0.80 \\
\hline 10 & 20.00 & 0.50 & 0.50 \\
\hline 11 & 20.00 & 1.25 & 0.20 \\
\hline 12 & 20.00 & 1.25 & 0.80 \\
\hline 13 & 20.00 & 2.00 & 0.50 \\
\hline
\end{tabular}

For each of the above parameters variability range was established arbitrarily and using three-level fractional plan 3(k-p) - Box-Behnken plan - number and cases of variability were determined, as given in Table 1 .

Method for determination of bending of transducer $w$, procedures related to description of fracture process and selected method of multi-object optimization are presented in the further part of this paper.

\subsection{Electromechanical characteristics of transducer}

Consecutive Eq. (1), used to determine electromechanical characteristics of transducer was developed using method (described in detail in papers $[13,14,21])$ involving implementation of two types of piezoelectric modules to a homogeneous beam: $P B S$ (piezoelectric bimorph segment) and PTS (piezoelectric triple segment), respectively. Taking into account structural variability, as described in chapter 2.1, and load conditions, general differential equation for strain of transducer can be noted as follows:

$$
\begin{aligned}
& \frac{\partial^{2} y}{\partial x^{2}}=\frac{M_{e B}}{E_{p} J_{o B}}\left(\begin{array}{l}
(H[x]-H[x-L])- \\
+H\left[x-x_{2}\right]+H\left[x-\left(x_{2}+L_{3}\right)\right]
\end{array}\right)+ \\
& +\frac{M_{e T}}{E_{p} J_{o T}}\left(H\left[x-x_{2}\right]-H\left[x-\left(x_{2}+L_{3}\right)\right]\right) .
\end{aligned}
$$

where: $H\left[x-x_{i}\right]$ is Heaviside's function; $J_{o B}, J_{o T}$ are averaged moments of inertia for piezoelectric segments; $M_{e B}$, $\mathrm{M}_{e T}$ are bending moments generated by piezoelectric segments.

Bending moment and averaged moment of inertia for PBS segments is calculated from Eqs. (2) and (3):

$$
\begin{aligned}
& M_{e B}=d_{31} E_{p}{ }^{2} V \beta, \\
& J_{o B}=\frac{b \beta_{2}}{12 E_{p}\left(E_{b} t_{2}+E_{p} t_{1}\right)},
\end{aligned}
$$

where: $\beta=\frac{6 E_{b} J_{o B} t_{2}\left(t_{2}+t_{1}\right)}{\beta_{2}}$,

$$
\beta_{2}=E_{b}{ }^{2} t_{2}^{4}+E_{p}^{2} t_{2}^{4}+2 E_{b} E_{p} t_{2} t_{1}\left(2 t_{2}^{2}+3 t_{2} t_{1}+t_{1}^{2}\right) .
$$

Equations (4) and (5) describe bending moment and averaged moment of inertia for PTS segment, respectively:

$$
\begin{aligned}
& M_{e T}=d_{31} E_{p}{ }^{2} V \theta, \\
& J_{o T}=\frac{b\left(\alpha_{1}+\alpha_{2}+\alpha_{4}\right)}{12 E_{p}\left(E_{b} t_{2}+E_{p}\left(t_{1}+t_{3}\right)\right)},
\end{aligned}
$$

where $d_{31}$ is piezoelectric constant:

$$
\begin{aligned}
& \alpha_{1}=E_{b}{ }^{2} t_{2}^{4}-2 E_{b} E_{p} t_{2}\left(t_{1}^{3}+t_{3}^{3}+t_{2}^{2}\left(t_{1}+t_{3}\right)+3 t_{2}\left(t_{1}^{2}+t_{3}^{2}\right)\right) \\
& \alpha_{2}=E_{p}{ }^{2}\left(t_{1}{ }^{4}-2 t_{1}{ }^{3} t_{3}+t_{3}{ }^{4}-6 t_{1}{ }^{2} t_{3}\left(2 t_{2}+t_{3}\right)-2 t_{1} t_{3}\left(6 t_{2}{ }^{2}+6 t_{2} t_{3}+t_{3}{ }^{2}\right), \alpha_{3}=E_{b}{ }^{2} t_{2}{ }^{4}+E_{p}{ }^{2}\left(t_{1}{ }^{4}+t_{1}{ }^{3} t_{3}+t_{1} t_{3}{ }^{3}+t_{3}{ }^{4}\right),\right. \\
& \alpha_{4}=6 E_{p}\left(E_{p} t_{1} t_{3}\left(t_{1}+2 t_{2}+t_{3}\right)^{2}+E_{b} t_{2}\left(t_{1}^{3}+2 t_{1}^{2} t_{2}+t_{1} t_{2}^{2}+t_{3}\left(t_{2}+t_{3}\right)^{2}\right)\right) \\
& \theta=\frac{-b J_{o T}\left(t_{1}+t_{3}\right)\left(t_{1}+2 t_{2}+t_{3}\right)\left(E_{b} t_{2}+E_{p}\left(t_{1}+t_{3}\right)\right)\left(E_{b} t_{2}^{3}+E_{p}\left(t_{1}^{3}+t_{3}^{3}\right)\right)}{2\left(E_{b} J_{b}+E_{p} J_{p 1}+E_{p} J_{p 3}\right)\left(t_{1}+t_{2}+t_{3}\right)\left(\alpha_{1}+\alpha_{2}\right)},
\end{aligned}
$$


$J_{b}=\frac{b t_{2}^{3}}{12}, J_{p 1}=\frac{b t_{1}^{3}}{12}, J_{p 2}=\frac{b t_{2}^{3}}{12}$ is moments of inertia for the individual layers.

By integrating the equation (1), in order to determine constants of integration using boundary conditions as follows:

$$
\frac{\partial y}{\partial x}(0)=0, y(0)=0
$$

electromechanical characteristics of transducer (7) was obtained:

$$
y(x)=\frac{d_{31} E_{p} A_{1} v}{2 J_{o B} J_{o T}},
$$

where:

$$
A_{1}=\left(\begin{array}{l}
\beta J_{o T} H[x] x^{2}- \\
-\left(\beta J_{o T}-\theta J_{o B}\right)\left(H\left[x-x_{2}\right]\left(x-x_{2}\right)^{2}+x\left(L_{3}+x_{2}\right)^{2}\right)+ \\
+H\left[x-L_{3}-x_{2}\right]\left(L_{3}-x+x_{2}\right)^{2}-\beta J_{o T} H\left[x-L_{1}\right]\left(L_{1}-x\right)^{2}
\end{array}\right) .
$$

Relative deflection of the transducer right end was calculated based on the following equation:

$$
w=\frac{y(L)}{L}
$$

\subsection{Conditions for fracture process initiation}

In order to define conditions, at the presence of which fracture process initiation will occur, it is necessary to develop a qualitative and quantitative description of singular stress fields occurring around structural notch-tip area (Fig. 2) with notch angle $\beta=90^{\circ}$, formed between lower piezoelectric element 3 (Fig. 1) and non-piezoelectric layer 2 (Fig. 1).
The nature of such stress fields is considerably different from those generated by notch located in a homogeneous material. Eigenvalue $\lambda$, determining the nature of singular stress fields, can have both complex and real values $[15,17,20]$. This creates difficulties in both analytical description of stress fields and formulation of adequate strength criteria [17]. Because stresses can be described by complex exponent $\lambda=\lambda_{r}+i \delta$, generalized stress intensity factors $K_{j}$ were defined as follows [17]:

$$
\left(\sigma_{\varphi}+\mathrm{i} \tau_{r \varphi}\right)_{\varphi=0}=\frac{K_{I}+i K_{I I}}{\sqrt{2 \pi} r^{1-\lambda_{r}}}\left(\frac{r}{2 a}\right)^{\mathrm{i} \delta} \cosh [\pi \delta] .
$$

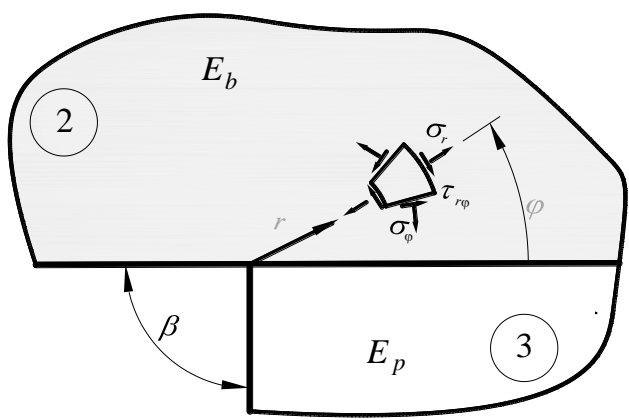

Fig. 2 Structural notch located on interface between non-piezoelectric element and lower piezoelectric layer

By using the eigenfunction expansion method [22, 17], relation (9) and below boundary conditions:

- of upper surface of structural notch, for $\varphi=\pi$;

$\sigma_{\varphi 2}=\tau_{r \varphi 2}=0$;

- of lower surface of structural notch, for $\varphi=-\pi / 2$;

$\sigma_{\varphi 3}=\tau_{r \varphi 3}=0$;

- along the interface, for $\varphi=0$;

$u_{r 2}=u_{r 3} ; u_{\varphi 2}=u_{\varphi 3} \sigma_{\varphi 2}=\sigma_{\varphi 3} ; \tau_{r \varphi 2}=\tau_{r \varphi 3} ;$,

analytical relations describing singular stress fields (10) in the interface plane and characteristic equation (11) can be determined [17].

$$
\begin{aligned}
\sigma_{\varphi 2,3 \varphi=0}= & \left.\frac{1}{\sqrt{2 \pi}} \sqrt{K_{I}{ }^{2}+K_{I I}{ }^{2}} r^{\lambda_{r}-1} \cos \left[\arctan \left[\frac{K_{I I}}{K_{I}}\right]+\delta \ln \left[\frac{r}{2 a}\right]\right] \cosh [\pi \delta]\right] \\
\tau_{r \varphi 2,3_{\varphi=0}}= & \left.\frac{1}{\sqrt{2 \pi}} \sqrt{K_{I}{ }^{2}+K_{I I}{ }^{2}} r^{\lambda_{r}-1} \sin \left[\arctan \left[\frac{K_{I I}}{K_{I}}\right]+\delta \ln \left[\frac{r}{2 a}\right]\right] \cosh [\pi \delta]\right], \\
& 2 \mu_{2}^{2} \sin ^{2}[\pi \lambda]\left(2 \kappa_{3} \cos [\pi \lambda]+\kappa_{3}^{2}-4 \lambda^{2}+1\right)-\mu_{3}^{2}\left(2 \lambda^{2}+\cos [\pi \lambda]-1\right)\left(2 \kappa_{2} \cos [2 \pi \lambda]+\kappa_{2}^{2}+1\right)+ \\
& +2 \mu_{2} \mu_{3} \sin ^{2}[\pi \lambda]\left(2\left(\kappa_{2} \kappa_{3}+1\right) \cos [\pi \lambda]-\left(\kappa_{2}-1\right)\left(\kappa_{3}+4 \lambda^{2}-1\right)\right)=0,
\end{aligned}
$$

where: $\mu_{i}=\frac{E_{i}}{2\left(1+v_{i}\right)}$-shear modulus $\kappa_{i}=\left(3-v_{i}\right) /\left(1+v_{i}\right)$ - a plane stress, $\kappa_{i}=\left(3-4 v_{i}\right)$ - a plane strain, $v_{i}$-Poisson's ratio,

$i=2,3$.

With obtained characteristic equation $(11)$ it is possible to determine enginevalues $\lambda\left(\lambda_{r}=\operatorname{Re}[\lambda], \operatorname{Im}[\lambda]=\delta\right)$.

Analytical description of stress fields for any angular coordinate $\varphi$ can be found, e.g. in paper [17].
For a quantitative description of stress fields it is necessary to determine generalized stress intensity factors $K_{j}$. They were determined based on a comparison of analyt- 
ical solutions to numerical solution FEM. Based on analytical description of stress fields Eq. (10) the following linear functions of coordinate $r$ Eq. (12) were formulated [17]:

$$
\begin{aligned}
& K_{I(r)}=\frac{\sqrt{2 \pi}\left(r_{n} r_{n+1}\right)^{1-\lambda_{r}} \operatorname{sech}[\pi \delta]}{r_{n}-r_{n+1}}\left(\begin{array}{l}
\left.r_{n}^{\lambda_{r}}\left(\sigma_{\left(r_{n+1}\right)} \cos \left[\delta \ln \left[\frac{r_{n+1}}{2 a}\right]\right]+\tau_{\left(r_{n+1}\right)} \sin \left[\delta \ln \left[\frac{r_{n+1}}{2 a}\right]\right]\right)\right) \\
\left.-r_{n+1} \lambda_{r}\left(\sigma_{\left(r_{n}\right)} \cos \left[\delta \ln \left[\frac{r_{n}}{2 a}\right]\right]+\tau_{\left(r_{n}\right)} \sin \left[\delta \ln \left[\frac{r_{n}}{2 a}\right]\right]\right)\right)
\end{array}\right) . \\
& \left.K_{I I(r)}=\frac{\sqrt{2 \pi}\left(r_{n} r_{n+1}\right)^{1-\lambda_{r}} \operatorname{sech}[\pi \delta]}{r_{n}-r_{n+1}}\left(\begin{array}{l}
\left.r_{n}^{\lambda_{r}}\left(\tau_{\left(r_{n+1}\right)} \cos \left[\delta \ln \left[\frac{r_{n+1}}{2 a}\right]\right]-\sigma_{\left(r_{n+1}\right)} \sin \left[\delta \ln \left[\frac{r_{n+1}}{2 a}\right]\right]\right)\right) \\
-r_{n+1}^{\lambda_{r}}\left(\tau_{\left(r_{n}\right)} \cos \left[\delta \ln \left[\frac{r_{n}}{2 a}\right]\right]-\sigma_{\left(r_{n}\right)} \sin \left[\delta \ln \left[\frac{r_{n}}{2 a}\right]\right]\right)
\end{array}\right)\right)
\end{aligned}
$$

Then, using data obtained from FEM solution $\sigma_{r i}, \tau_{r i}$, set of function values Eq. (12) (for selected points with coordinates $r$ is located around corner-tip area) was determined. This enabled, using linear regression Eq. 13), to determine generalized stress intensity factors for $r=0$.

$$
\left.\begin{array}{c}
K_{I}=K_{I(r=0)}=\frac{\sum_{n=1}^{n+1} K_{I(r)} \sum_{n=1}^{n+1} r_{n}^{2}-\sum_{n=1}^{n+1} r_{n} \sum_{n=1}^{n+1} K_{I(r)} r_{n}}{(n+1) \sum_{n=1}^{n+1} r_{n}^{2}-\left(\sum_{n=1}^{n+1} r_{n}\right)^{2}} \\
K_{I I}=K_{I I(r=0)}=\frac{\sum_{n=1}^{n+1} K_{I I(r)} \sum_{n=1}^{n+1} r_{n}^{2}-\sum_{n=1}^{n+1} r_{n} \sum_{n=1}^{n+1} K_{I I(r)} r_{n}}{(n+1) \sum_{n=1}^{n+1} r_{n}^{2}-\left(\sum_{n=1}^{n+1} r_{n}\right)^{2}}
\end{array}\right\} .
$$

As mentioned, to determine the $K_{j}$ factors it was required to carry out numerical calculations. Therefore, using FEM (ANSYS) [23], the transducer was modelled as a plane strain problem. Layers of the transducer were circumscribed with quadrangle, eight-node finite elements with increased refinement around the notch-tip area, with triangular special elements surrounding the singular point [24]. Two types of finite elements were used: PLANE223-piezoelectric material, PLANE82-beam. In simulations carried out, geometrical and material parameters were changed - in accordance with Table 1.

For determination of conditions for fracture process initiation, it is required to develop an adequate criterion. This criterion should include accurately determined equation with defined criterion parameters, on basis of which it is possible to predict the moment of fracture process initiation. Paper [17] shows that selection of the criterion should be dependant on exponent $\lambda$. If the enginevalue $\lambda$ takes complex values (between $0 \div 1$ ), satisfactory results of bi-material structure with structural notch strength prediction are obtained using the Novozhilov hypothesis. This hypothesis assumes that the fracture process will be initiated when damage function $-f\left(\sigma_{\varphi 2,3 \varphi=0}, \tau_{r \varphi 2,3 \varphi=0}, \sigma_{r 2 \varphi=0}\right)$ reaches the criterion value. If the enginevalue is a real number, the best results of the prediction are obtained using a criterion based on stress intensity factor.

Therefore, based on the characteristic Eq. (11) value of exponent $\lambda$ was determined at first. Based on ob- tained solution, it can be claimed that parameter $\lambda$ for investigated structural notch always takes real values between $0 \div 1(\operatorname{Im}[\lambda]=\delta=0)$. Thus to determine the moment of fracture process initiation, criterion Eq. (14) was used, based on generalized stress intensity factor:

$$
K^{n}=K_{\text {Ic }},
$$

where: $K_{\text {Ic }}$ is fracture toughness, $K^{n}$ is normalized, generalized stress intensity factor.

It follows that fracture process will be initiated when factor $K^{n}$ reaches the criterion value $K_{\text {Ic }}$ (material constant). It is obvious that susceptibility of piezoelectric transducer to fracture will be lower if the calculated factor $K^{n}$ takes as low values as possible. Normalized, generalized stress intensity factor can be calculated from the following equation [25]:

$$
K^{n}=\frac{\sigma_{o}}{2}\left(\frac{\left|K_{\mathrm{I}}\right|}{\lambda_{\mathrm{r}} \sigma_{o}}\right)^{\frac{1}{2\left(1-\lambda_{\mathrm{r}}\right)}}
$$

where: $K_{I}$ is generalized stress intensity factor Eq. (13), $\lambda_{r}$ is enginevalue determined from characteristic Eq. (11), $\sigma_{o}$ is stress at which factor $K_{I}$ was calculated.

Stress $\sigma_{o}$, induced by the applied voltage $V$, can be calculated from Eq. (16):

$$
\sigma_{o}=\frac{M_{e T} y_{c}}{J_{o T}},
$$

where: $y_{c}=\frac{2 t_{2} t_{1}+\left(t_{2}+t_{3}\right)^{2}+\frac{E_{b}}{E_{p}} t_{2}\left(t_{2}+2 t_{3}\right)}{2\left(\frac{E_{b}}{E_{p}} t_{2}+t_{1}+t_{3}\right)}$. is the coordinate of $\mathrm{s}$ the centre of gravity of the cross-section area.

\subsection{Grey relational analysis method}

To determine optimal parameters related to geometry and stiffness of individual components of piezoelectric transducer under analysis, a method of multi-object optimization - GRA (Grey Relational Analysis) was selected. This 
method is used to determine a relation between selected factors which in overall influence qualitative and quantitative characteristics of the process under research. With this, it is possible to select, from experimental data, the best combinations of these factors and formulate a prognostic function. The use of this approach has been positively verified in many areas, such as agriculture, economy or arms industry $[26,27]$. Detailed description of this method can be found, e.g. in paper [28]. In GRA analysis three main stages can be distinguished:

I. standardization of experimental data SD;

II. determination of the Grey Relational Coefficient (GRC);

III. determination of the Grey Relational Grade (GRG).

Purpose of the stage $I$ is to standardize the input data. Standardization is used when data under analysis is expressed in various units and large range of their variability occurs. If the expectancy is the lower - the-better, then the standardizing function can be expressed by Eq. (17). If the expectancy is, however, the higher - the-better, then it can be expressed by Eq. (18) [28]:

$$
\begin{aligned}
& x_{i}^{*}(k)=\frac{\max x_{i}^{o}(k)-x_{i}^{o}(k)}{\max x_{i}^{o}(k)-\min x_{i}^{o}(k)}, \\
& x_{i}^{*}(k)=\frac{x_{i}^{o}(k)-\min x_{i}^{o}(k)}{\max x_{i}^{o}(k)-\min x_{i}^{o}(k)},
\end{aligned}
$$

where: $i=1, \ldots m ; k=1, \ldots n, m$ is number of experimental data items, $n$ is the number of parameters;

$x_{i}^{o}(k)$ is original experimental data,

$x_{i}^{*}(k)$ is the experimental data after data standardization,

$\max x_{i}^{o}(k), \min x_{i}^{o}(k)$ are the largest and the smallest values of $x_{i}^{o}(k)$.

In the issue under analysis $x_{i}^{o}(1)=w_{i}$ (relative deflection) and $x_{i}^{o}(2)=K_{i}^{n}$ (normalized, generalized stress intensity factor). According to the assumption that the largest deflection is expected, data standardization $x_{i}^{o}(1)$ was carried out by Eq. (18). Equation (17) was applied for data

$x_{i}^{o}(2)$ - expected lowest values of normalized stress intensity factor.

The Grey Relational Coefficient (GRC) [28] is calculated by Eq. (19):

$$
\xi_{i}(k)=\frac{0.5}{1.5-x_{i}^{*}(k)} .
$$

After GRC calculation the Grey Relational Grade GRG Eq. (20) is calculated:

$$
\gamma_{i}=\frac{1}{n} \sum_{k=1}^{n} \xi_{i}(k)
$$

The GRG coefficient defines compatibility of the tested parameter (in the analysed issue for parameters $w_{i}$ and
$K_{i}^{n}$ ) with factors affecting the quantity value of this parameter (relative stiffness $-e$, relative height $-t$, relative lengthl). The GRG Eq. (20) coefficient takes values between 0 and 1. The highest the value the biggest the compatibility, and factors affecting the $w_{i}$ and $K_{i}^{n}$ more optimal (desired). It is assumed that for $\gamma_{i}>0.6$, influence of factors on parameter under investigation is significant [28].

\section{Test results}

As already mentioned purpose of tests carried out was to determine an optimum relation between stiffness and geometric characteristics of individual components of piezoelectric transducer, for which deflection of the right end of the transducer would be greatest at the greatest resistance to fracture at the same time. Therefore, for the below data:

- $\quad$ piezoelectric strain coefficients: $d_{31}=2.2^{\prime} 10^{-11}$ $\mathrm{C} / \mathrm{N}, d_{32}=0.3^{\prime} 10^{-11} \mathrm{C} / \mathrm{N}, d 33=-3.0^{\prime} 10^{-11} \mathrm{C} / \mathrm{N}$;

- $\quad$ relative permittivity at constant stress: $\left(\varepsilon_{33}\right)^{\mathrm{T}}=12$;

- Poisson's ratio: $v_{p}=v_{b},=0.29$;

- Young's modulus: $E_{p}=2.0^{\prime} 10^{9}, E_{b}=e E_{p}, \mathrm{~N} / \mathrm{m}^{2}$;

- layers thickness: $t_{1}=0.002, t_{2}=0.001, t_{3}=t t_{p}, \mathrm{~m}$;

- $\quad$ beams length $L=0.06, L_{3}=l L, \mathrm{~m}$;

- electrode voltage $V=-100$ Volts.

The relative deflection Eq. (8), normalized, generalized stress intensity factor Eq. (15) were determined, and, according to method described in chapter 2.4, optimization procedures were carried out.

The obtained results are given in Table 2. Optimization parameters $-e, t, l$ - were determined for 13 tests based on Table 1.

For the constructional variant, if lower piezoelectric element is located in the centre, within transducer structure two notches will occur - on both sides of lower piezoelectric layer. Therefore coefficients $K_{i}^{n}$ were calculated for both notches. As should be expected the obtained values were virtually identical. Thus in Table 2 data for only one notch (left one) are given.

As already mentioned in chapter 2.4 the GRA method enables:

- selection from among the data obtained from experimental testing, the most optimal cases [29];

- formulation of prognostic function.

To select the most favourable geometrical and material parameters for transducer it is necessary to calculate average values GRG for all levels of optimized parameters - e,t,l. As an optimal case variant corresponding to maximum value of averaged GRG for a given level is assumed. In experimental tests carried out, three levels of each particular parameter were used, as shown in Table 3.

Averaged values GRG for particular levels were calculated from the equation below:

$$
\tilde{\gamma}_{e, l, t_{j}}=\frac{1}{n} \sum_{1}^{n} \gamma_{i}
$$

where: $j=1,2,3$ is level of parameter, $n$ is number of experimental data for level $j$. 
Optimized parameters and optimization results

\begin{tabular}{|c|c|c|c|c|c|c|c|}
\hline Case no. & $w_{i}=x_{i}^{o}(1)$ & $K_{i}^{n}=x_{i}^{o}(2), \mathrm{Pa} \mathrm{m}^{0.5}$ & $\begin{array}{c}x_{i}^{*}(1) \\
\mathrm{SD}\end{array}$ & $\begin{array}{c}x_{i}^{*}(2) \\
\mathrm{SD}\end{array}$ & $\begin{array}{c}\xi_{i}(1) \\
\text { GRC }\end{array}$ & $\begin{array}{c}\xi_{i}(2) \\
\text { GRC }\end{array}$ & $\begin{array}{c}\gamma_{i} \\
\text { GRG }\end{array}$ \\
\hline 1 & $1.884^{\prime} 10^{-5}$ & 62.09 & 0.777778 & 0.932239 & 0.692308 & 0.880652 & 0.78648 \\
\hline 2 & $1.385^{\prime} 10^{-5}$ & 4.69 & 0.417749 & 1 & 0.462 & 1 & 0.731000 \\
\hline 3 & $1.154^{\prime} 10^{-5}$ & 5.08 & 0.251082 & 0.99954 & 0.400347 & 0.99908 & 0.699713 \\
\hline 4 & $1.113^{\prime} 10^{-5}$ & 17.16 & 0.221501 & 0.985279 & 0.391084 & 0.9714 & 0.681242 \\
\hline 5 & $1.727^{\prime} 10^{-5}$ & 607.2 & 0.664502 & 0.28873 & 0.598446 & 0.41279 & 0.505618 \\
\hline 6 & $2.192^{\prime} 10^{-5}$ & 605.10 & 1 & 0.291209 & 1 & 0.413636 & 0.706818 \\
\hline 7 & $1.351^{\prime} 10^{-5}$ & 56.83 & 0.393218 & 0.938448 & 0.45176 & 0.89039 & 0.671075 \\
\hline 8 & $1.365^{\prime} 10^{-5}$ & 36.15 & 0.403319 & 0.962861 & 0.455921 & 0.930858 & 0.693389 \\
\hline 9 & $0.806^{\prime} 10^{-5}$ & 32.47 & 0 & 0.967205 & 0.333333 & 0.938448 & 0.635891 \\
\hline 10 & $2.166^{\prime} 10^{-5}$ & 851.78 & 0.981241 & 0 & 0.963839 & 0.333333 & 0.648586 \\
\hline 11 & $1.372^{\prime} 10^{-5}$ & 8.31 & 0.408369 & 0.995727 & 0.45803 & 0.991526 & 0.724778 \\
\hline 12 & $1.289^{\prime} 10^{-5}$ & 91.63 & 0.348485 & 0.897366 & 0.434211 & 0.829691 & 0.631951 \\
\hline 13 & $1.006^{\prime} 10^{-5}$ & 53.11 & 0.1443 & 0.94284 & 0.368813 & 0.897408 & 0.63311 \\
\hline
\end{tabular}

Table 3

Applied levels of optimized parameters

\begin{tabular}{|c|c|c|c|}
\hline Parameter & level 1 & level 2 & level 3 \\
\hline$e$ & 1 & 10.5 & 20 \\
\hline$t$ & 0.5 & 1.25 & 2 \\
\hline$l$ & 0.2 & 0.5 & 0.8 \\
\hline
\end{tabular}

Apart from selection of optimal geometrical and material parameters, also it is possible to determine which optimized parameter has the greatest influence on GRG value. The greatest influence has the parameter for which the difference between minimum and maximum value GRG is the greatest Eq. (22):

$$
\Delta G R G_{e, l, t}=\max \left(\tilde{\gamma}_{e, l, t}\right)-\min \left(\tilde{\gamma}_{e, l, t}\right)
$$

Table 4 and Fig. 3 show averaged GRG values for individual levels calculated from Eq. (21).

Table 4

Average GRG values Eq. (21) for individual levels

\begin{tabular}{|c|c|c|c|c|}
\hline \multirow{2}{*}{$\begin{array}{c}\text { Param- } \\
\text { eter }\end{array}$} & \multicolumn{3}{|c|}{$\tilde{\gamma}_{e, l, t_{j}}$} & \multirow{2}{*}{$\Delta G R G_{e, l, t}$} \\
\cline { 2 - 5 } & level 1 & level 2 & level 3 & \\
\hline$e$ & $0.724609 *$ & 0.642558 & 0.659606 & $0.082051 * *$ \\
\hline$t$ & 0.661875 & $0.691703 *$ & 0.660908 & 0.030795 \\
\hline$l$ & 0.663696 & $0.684099 *$ & 0.668593 & 0.020402 \\
\hline \multicolumn{5}{|c}{$*_{-}$max $G R G$ (the optimal variant) } \\
\multirow{2}{*}{$* *$ max $\Delta G R G_{e, j, t}$ (parameter with the greatest influence) } \\
\hline
\end{tabular}

Example calculation of average GRG value (for level of the first parameter $e$ ) and $\triangle G R G_{e}$ is given below: $\gamma_{e_{1}}=\frac{1}{4}(0.78648+0.73100+0.69971+0.68124)=0.724609$,

$\Delta G R G_{e}=0.724609-0.642558=0.082051$.

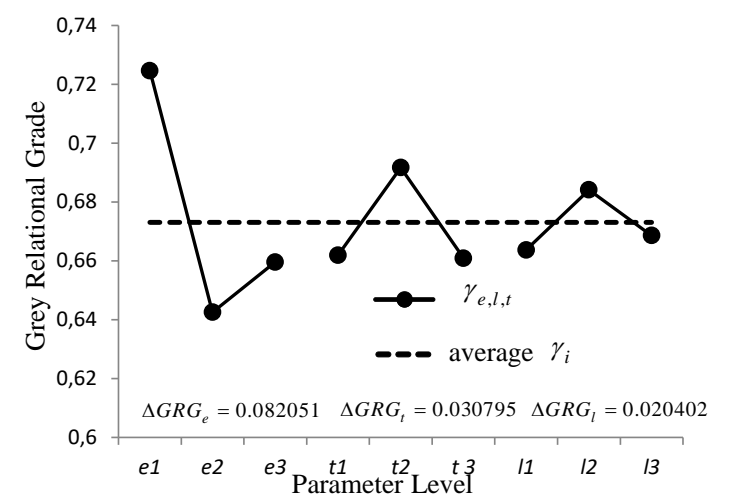

Fig. 3 Average GRG values Eq. (21) for individual levels

Through analysis of data given in Table 4 and Fig. 3 it can be claimed that among experimental tests carried out, the best constructional variants of the analysed transducer were achieved for the following levels of optimized parameters: $e=1, t=1.25, l=0.5$.

Moreover, when comparing parameters $\triangle G R G_{e, l, t}$, it is evident that factor that has the greatest influence on GRG, and thus on the strength and deflection of the transducer, is relative stiffness $e$.

Apart from selection of optimal parameters obtained from experimental tests, as already mentioned, the GRA method also allows for prediction. The sought prognostic function $\operatorname{GRG}(e, t, l)$ can be formulated as Eq. (22):

$$
\begin{aligned}
& \operatorname{GRG}(e, t, l)=A_{0}+A_{1} e+A_{2} e^{2}+ \\
& +C_{1} l+D_{2} e l+C_{2} l^{2}+B_{1} t+ \\
& +D_{1} e t+D_{3} l t+B_{2} t^{2} .
\end{aligned}
$$


Constants $A_{i}, B_{i}, C_{i}, D_{i}$ were determined by nonlinear regression method (estimation method - Gauss-Newton, loss function-method of least squares, level of confidence $p=95 \%$ ). In statistical analyses the used arguments $e, t, l$ - given in Table 1 and dependent variable GRG - Table 2. Obteined constants $A_{i}, B_{i}, C_{i}, D_{i}$ are given bellow: $A_{0}=0.485517, A_{1}=-0.013700, A_{2}=0.000431$,

$B_{1}=0.210344, B_{2}=-0.040135, C_{1}=0.569369$,

$C_{2}=-0.145221, D_{1}=0.0031495, D_{2}=-0.005398$,

$D_{3}=-0.287444, R^{2}=0.9970$.

Fig. 4 provides comparison of limit values of GRG parameters obtained from experimental (simulated) tests carried out and values calculated from Eq. (22) (predicted).

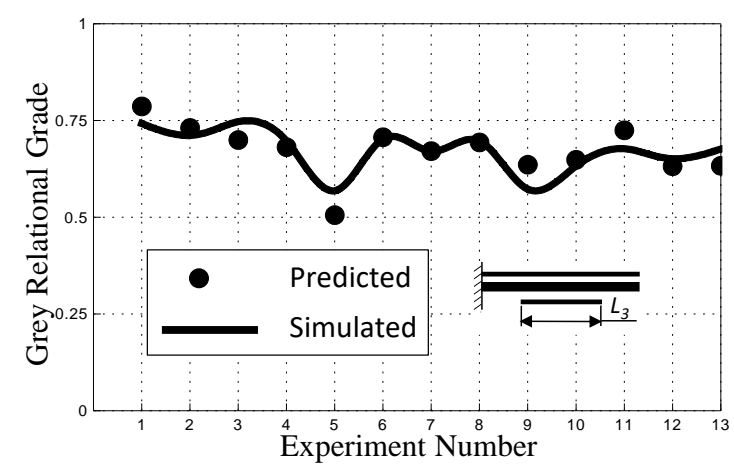

Fig. 4 Values of GRG coefficients - determined (Table 2) and predicted Eq. (22)

By Eq. (22) it is possible to determine such geometrical-material parameters so as to obtain the desired value of GRG parameter (best if near to 1). Fig. 5 depicts the range of variability of optimized parameters $-e, t, l-$ for which the GRG value is greater than arbitrarily selected value -0.71 .

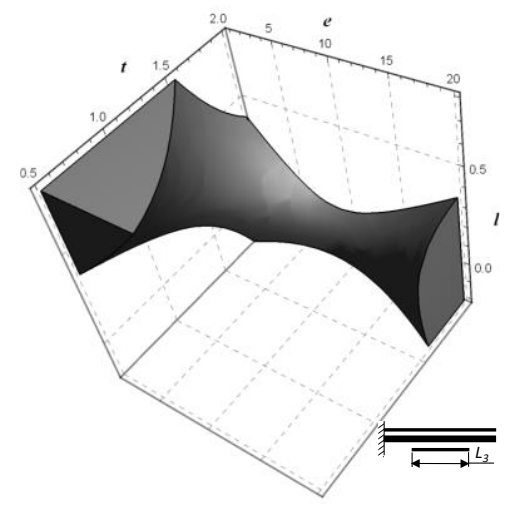

Fig. 5 Values of coefficient GRG $(e, t, l) \geq 0.71$ calculated from Eq. (22)

Through analysis of the above figure it can be claimed that the transducer in a design where piezoelectric element is located in the centre, is characterized by the greatest flexibility in selection of geometrical and material parameters.

\section{Conclusions}

Using the GRA method the optimization of the three-layer piezoelectric transducer was made. The best design variants were selected $-e=1$ (relative stiffness), $t=1.25$ (relative height), $l=0.5$ (relative length). What is more, it was claimed that the factor having the greatest influence on deflection and strength of transducer is relative stiffness $e$. In addition, the prognostic function, allowing to determine the optimal parameters related to the geometry and stiffness of individual components of the piezoelectric converter, for which the $K^{n}$ function assumes the smallest values with the highest relative deflection simultaneously, was proposed. Based on this function, it can be concluded that there is a great flexibility in a selection of geometrical and material parameters which will assure optimal strength and utility features.

\section{Acknowledgements}

Studies were carried out within work no. S/WM/1/18 and funded from under development of science by Ministry of Science and Higher Education.

\section{References}

1. Curie, P.J.; Curie J. 1880. Crystal Physics-Development by Pressure 0/ Polar Electricity in Hemihedral Crystals with Inclined Faces, Acad. Sci.(Paris) C. R. Hebd. Seances 91: 294 (in French).

2. Berlincourt, D. A.; Curran, D. R.; Jaffe, H.; Mason, W. P. 1964. Physical Acoustics, Principles and Methods, 1, A, Academic Press, New York. http://dx.doi.org/10.1126/science.145.3639.1424-a.

3. Busch-Vishniac, I.J. 1999. Electromechanical Sensors and Actuators, Springer-Verlag. http://dx.doi.org/10.1007/978-1-4612-1434-2.

4. Smits, J.G.; Dalke, S.I.; Cooney, T.K. 1991. The constituent equations of piezoelectric bimorphs, Sensors and Actuators A, 28: 41-61.

http://dx.doi.org/10.1016/0924-4247(91)80007-C.

5. Wang, Q.; Cross, L.E. 1999. Constitutive equations of symmetrical triple-layer piezoelectric benders, IEEE Trans. Ultrason. Ferroelec.Freq. Contr. 46: 1343-1351. http://dx.doi.org/10.1109/58.808857.

6. Ballas, R.G.; Schlaak, H.F.; Schmid, A. J. 2006. The constituent equations of piezoelectric multilayer bending actuators in closed analytical form and experimental results, Sens. Actuators A: Phys. 130-131 91-98. https://doi.org/10.1016/j.sna.2005.11.034.

7. Xiang, H.J.; Shi, Z.F. 2008. Static analysis for multilayered piezoelectric cantilevers, International Journal Of Solids and Structures 45(1): 113-128. http://dx.doi.org/10.1016/j.ijsolstr.2007.07.022.

8. Edqvist, E.; Hedlund, E.; Lundberg, B. 2010. Quasistatic and dynamic electromechanical response of piezoelectric multilayer cantilever beams, Actuat. A 157: 198-209. https://doi.org/10.1016/j.sna.2009.11.013.

9. Ballas, R.G. 2007. Piezoelectric Multilayer Beam Bending Actuators: Static and Dynamic Behavior and Aspects of Sensor Integration, Springer-Verlag, Berlin,Heidelberg.

10. Park J.K.; Moon, W.K. 2005. Constitutive relations for piezoelectric benders under various boundary conditions, Sens. Actuat. A 117: 159-167. https://doi.org/10.1016/j.sna.2004.03.051.

11. Raeisifard, H.; Bahrami, M.N.; Yousefi-Koma, A.; Fard, H. R. 2014. Static characterization and pull-in voltage of a micro-switch under both electrostatic and 
piezoelectric excitations, European Journal of Mechanics A/Solids 44: 116-124 https://doi.org/10.1016/j.euromechsol.2013.10.012.

12. Dunsch, R.; Breguet, J.M. 2006. Unified mechanical approach to piezoelectric bender modelling, Sens. Actuators A: Phys. 134(2): 436-446. https://doi.org/10.1016/j.sna.2006.06.033.

13. Mieczkowski, G. 2016. Electromechanical characteristics of piezoelectric converters with freely defined boundary conditions and geometry, Mechanika 22(4): 265-272. http://dx.doi.org/10.5755/j01.mech.22.4.12764.

14. Mieczkowski, G. 2017. The constituent equations of piezoelectric cantilevered three-layer actuators with various external loads and geometry, Journal of Theoretical and Applied Mechanics 55(1): 69-86 http://dx.doi.org/10.15632/jtam-pl.55.1.69.

15. Bogy, D.B. 1971. Two edge-bonded elastic wedges of different materials and wedge angles under surface tractions, Journal of Applied Mechanics 38: 377-386. http://dx.doi.org/10.1115/1.3408786.

16. Mirsayar, M.M.; Aliha, M.R.M.; Samaei, A.T. 2014. On fracture initiation angle near bi-material notches Effects of first non-singular stress term, Engineering Fracture Mechanics 119: 124-131. http://dx.doi.org/10.1016/j.engfracmech.2014.02.017.

17. Mieczkowski, G. 2017. Stress fields and fracture prediction for adhesively bonded bi-material structure with sharp notch located on the interface, Mechanics of Composite Materials, 53(3): 305-320. http://dx.doi.org/10.1007/s11029-017-9663-y.

18. Chen, M. C.; Ping, X.C.; Xie, H.M.; Liu, Z.W. 2008. Numerical and experimental analyses of singular electro-elastic fields around a $\mathrm{V}$-shaped notch tip in piezoelectric materials, Engineering Fracture Mechanics 75: 5029-5041. http://dx.doi.org/10.1016/j.engfracmech.2008.05.011.

19. Li, C.; Man, H.; Song, C.; Gao, W. 2013. Analysis of cracks and notches in piezoelectric composites using scaled boundary finite element method, Composite Structures 101: 191-203. http://dx.doi.org/10.1016/j.compstruct.2013.02.009.

20. Cheng, C.Z.; Yao, S.L.; Han, Z.L.; Recho, N.; Niu, Z.R. 2015. Evaluation of the singularity exponents and characteristic angular functions for piezoelectric Vnotches under in plane and out of plane conditions, Theoretical and Applied Fracture Mechanics 76: 50-59. http://dx.doi.org/10.1016/j.tafmec.2015.01.001.

21. Mieczkowski, G. 2017. The constituent equations of piezoelectric three-layer converters with different thickness and length of piezoelectric components, Mechanika 2017: Proceedings of the 22nd International Scientific Conference, 252-257.

22. Parton, V.Z. ; Perlin, P.I. 1984. Mathematical Methods of the Theory of Elasticity, Mir Publishers, Moscow.

23. Documentation for ANSYS, Coupled-Field Analysis Guide. 2010.

24. Tracey, D. M. 1971. Finite elements for determination of crack tip elastic stress intensity factors, Engineering Fracture Mechanics, 3(3): 255-265. http://dx.doi.org/10.1016/0013-7944(71)90036-1.

25. Seweryn, A,; Lukaszewicz, A. 2002. Verification of brittle fracture criteria for elements with V-shaped notches, Engineering Fracture Mechanics, pp. 6914871510 . http://dx.doi.org/10.1016/S0013-7944(01)00138-2.

26. Kung, C. F.; Wen, K. L. 200. Applying GRA and Grey Decision Making to evaluate the relationship between company attributes and its financial performance- A case study of ventures capital inTaiwan, Decision Support Systems 43: 842-852.

http://dx.doi.org/10.1016/j.dss.2006.12.012.

27. Tosun, N. 2006. Determination of optimum parameters for multi performance characteristics in drilling by using grey relational analysis, Int $\mathrm{J}$ Adv Manuf Techno.1 28:450-455. http://dx.doi.org/10.1007/s00170-004-2386-y.

28. Deng, J.L. 1989. Introduction to grey system. Journal of Grey System 1:1-24.

29. Taguchi, G. 1993. Taguchi methods: Design of experiments.Dearborn,MI, USA: American Supplier Institute Inc.

\section{G. Mieczkowski}

OPTIMIZATION AND PREDICTION OF DURABILITY AND UTILITY FEATURES OF THREE-LAYER PIEZOELECTRIC TRANSDUCERS

S u m m a r y

The paper presents the results of research related to the optimization and predicting of durability and utility features of three-layer piezoelectric transducers (two piezoelectric and one non-electric piezoelectric layers). The converter, in which one of the piezoelectric layers had a shorter length than the other two and was located in the middle of the transducer, has been analyzed (Fig.1). For such a construction of the transducer, the structural notch occurs at the ends of the shorter layer. In the notch tip area, there is a local stress concentration, which causes initiating of fracture process. Therefore, in the presented paper examined the impact of such factors as the relative stiffness $(e)$ and thickness $(t)$ of individual components and the dimensionless length $(l)$ of the shorter piezoelectric layer on:

- value of the proposed $K^{n}$ function specifying the conditions for initiating the cracking process;

- value of the relative deflection of the end of the transducer.

What is more, using Gray Relational Analysis Method, special functions $-f(e, t, l)$ also were developed and these enable prediction of functional and strength parameters for such transducer.

Keywords: piezoelectric transducer, deflection, electromechanical characteristics, fracture toughness.

Received April 20, 2018 Accepted June 14, 2018 